Recepción: 01 / 03/ 2018

Aceptación: 15 / 05 / 2018

Publicación: 07 / 07 / 2018

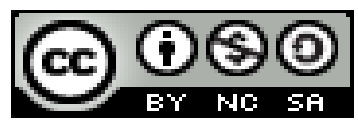

Ciencias Sociales

Artículo de Investigación

\title{
Modelo de Innovación para Incubación de negocios PYME’S, a partir de la actividad empresarial
}

\author{
Innovation Model for Business Incubation SMEs, \\ based on business activity
}

\author{
Modelo de Inovação para Incubação de \\ Empresas PME, com base na atividade \\ comercial
}

\author{
Miguel Á. Jaime-Baque ${ }^{\mathrm{I}} \dagger$ \\ angel.m.86@hotmail.com
}

Narda P. Merchán-Ponce II

narda.merchan@unesumedu.ec

Amparo B. Baque-Morán III

amparo.baque@unesum.edu.ec

\author{
Marina B. Alvarado-Chancay IV \\ marina_b@hotmail.es
Erick G. Salazar-Ponce V
erick.salazar@unesum.edu.ec

Viviana del Rocío Saltos-Burí VI

bibi_buri@hotmail.com

Correspondencia: angel.m.86@hotmail.com

I. Magister en Contabilidad y Auditoría, Ingeniero Comercial Especialización Comercio Exterior e Ing. en Medio Ambiente, Docente Contratado de la Universidad Estatal del Sur de Manabí, Facultad de Ciencia Económicas, Carrera de Auditoría, Jipijapa, Ecuador.

II. Master Administración de Empresas. Diplomado en Autoevaluación y Acreditación Universitaria, Economista, Docente Titular Auxiliar de la Universidad Estatal del Sur de Manabí, Facultad Ciencias Económicas, Carrera de Auditoría, Jipijapa, Ecuador.

III. Magister en Docencia Universitaria e Investigación Educativa, Diplomado en Autoevaluación y Acreditación Universitaria, Licenciada en Ciencias de la Educación Especialidad Comercio y Administración, Docente Titular Principal de la Universidad Estatal del Sur de Manabí, Facultad Ciencias Económicas, Carrera en Gestión Empresarial, Carrera de Administración de Empresas, Comisión Académica y Comisión de Investigación de la Carrera de Gestión Empresarial y Administración de Empresas, Jipijapa, Ecuador.

IV. Ingeniera en Computación y Redes, Docente Contratada de la Universidad Estatal del Sur de Manabí, Facultad de Ciencias Básicas en la Unidad de Nivelación y Admisión en el Área de Administración y el Área de Agricultura. Actualmente en el desarrollo de la tesis de la Maestría en Educación Informática, Jipijapa, Ecuador.

v. Doctor $(\mathrm{PhD})$. En Administración, Magister en Gerencia Educativa, Economista, Docente Titular Principal Carrera Administración de Empresas, Facultad de Ciencias Económicas Universidad Estatal del Sur de Manabí, Jipijapa, Ecuador.

VI. Magister en Contabilidad y Auditoría, Ingeniera en Administración de Empresas Agropecuarias. Docente Contratada de la Universidad Estatal del Sur de Manabí, Facultad Ciencias Económicas, Carrera de Administración de Empresas, Jipijapa,

Ecuador. 
Miguel Á. Jaime-Baque; Narda P. Merchán-Ponce; Amparo B. Baque-Morán; Marina B. Alvarado-Chancay; Erick G. Salazar-Ponce; Viviana del Rocío Saltos-Burí

\section{Resumen}

La finalidad del trabajo, es presentar un modelo de Gestión de incubación de negocios como sustento del desarrollo y crecimiento de las PYME's en Jipijapa, considerando como eje principal la vinculación entre Universidad, Empresa y Estado, para lograr un nivel de posicionamiento de gran impacto en la comunidad, Por lo que se parte del análisis de diferentes estudios empíricos y exploratorios de modelos de innovación de gran relevancia en otros países, que se están aplicando y han obtenido resultados eficientes en la actividad empresarial. Es por eso que la herramienta primordial para potenciar el desarrollo económico es la incubación de negocios, a través de un modelo de gestión que requieren las pequeñas y medianas empresas con nuevos enfoques innovadores, holísticos y dialéctica de transformación, para que el empresario tenga una cultura innovadora al interior de su empresa u organización. Como resultado, se diseñó un modelo de innovación para incubación de negocios PYME’s, a partir de la actividad empresarial, lo que se busco con este estudio es convertir un negocio pequeño en uno mucho más grande para impulsar el desarrollo económico, la generación de empleos en el país y sobre todo el avance en el conocimiento y la gestión empresarial.

Palabras clave: Desarrollo, Crecimiento, Transformación, Holística, Dialéctica. 


\section{Abstract}

As a reminder of accounting, from a different perspective of higher education, is to analyze the current competitive world of educational provision, where markets are increasingly global to the requirement, which are typical of the activities of academia, where shares experience and becomes part of the university community. This paper illustrates the importance of significant changes in how to teach and apply holistic and technological innovation in higher education, with an emphasis on cost accounting, which is the determination, recording and evaluation of internal results in the organization and the cost management is the precision and accuracy in the determination of the cost, oriented in the control of the management of the company and in the vision towards the environment, supported by actions of transformation and improvement of the quality of the System of Higher Education. The methodological approach is a documentary review of the exploratory theme, shows the situation that prevails in education and how technology and holistic innovation offer a real opportunity for higher education to include them as a strategic tool in the academic work.

Keywords: Development, Growth, Transformation, Holistic, Dialectic. 


\section{Introducción.}

En los últimos años el mundo ha evolucionado, se ha tornado como un espiral de la conciencia que gira alrededor de un inicio y un fin, es decir cada día las estructuras organizacionales son complejas, porque son rápidos y significativos los cambios de innovación tecnológica introducidos en las organizaciones, exige a las empresas estar preparados para competir a nivel nacional e internacional, es por eso que las empresas se han vuelto más agresivas en lo referente a innovación, perfeccionamiento continuo y reducción de costos. De hecho la contabilidad y gestión de costos, ha evolucionado dejando de lado lo tradicional adoptando nuevas posiciones de cambios innovativos en la educación superior, y el reto está en instaurar ventajas competitivas, maximizando la productividad y potencialidad del capital intelectual. Estas tendencias cambian el modo de innovar y organizar los negocios, así como el tipo de información que los empresarios requieren para planificar, dirigir y controlar las operaciones de sus empresas.

Todo esto, es lo que hace imprescindible un cambio en el trabajo docente educativo, mediante la inteligencia espiritual y la capacidad de aprender a ser feliz, porque se vive un cambio caracterizado por los acontecimiento de un nuevo paradigma global de nuestra ciencia que es la innovación holística y tecnológica, esto es lo que nos conlleva a necesitar una nueva educación, que sea una alternativa real para solucionar creativamente los problemas de la sociedad industrialcientífica y eliminar la visión del mundo mecanicista que todavía está presente.

En las universidades no se está aplicando lo holístico, las clases son controladas y no existe la libertad para trabajar en equipo y dejar desarrollar sus propias ideas y creatividad de los estudiantes, ya que esto es educación holística. Tanto es así, que la importancia de estos cambios son tan relevantes, el aprendizaje más importante es aprender a SER....aprender a ser es aprender a ser 
feliz, la educación de innovación holística y tecnológica parte de las potencialidades de los estudiantes sobre los conocimientos básicos de la contabilidad, para generar un enfoque integro, considerando lo emocional, intelectual, biológico, físico y espiritual, porque con este nuevo enfoque, el estudiante abre su mente de ese bagaje de conocimiento, creatividad y genialidad, para generar nuevos conceptos entre lo estudiado.

Desde esta perspectiva, la educación superior es considerada como un sistema de constante progreso y evolución. Los principios holísticos de interdependencia, diversidad, totalidad, flujo, cambio, unidad, sostenibilidad, entre otros, están en la base de este paradigma educativo, cuyo objetivo es analizar la importancia de la Innovación holística, tecnológica en el aprendizaje de la contabilidad y gestión de costos en la educación superior, explicando sus características más relevante en la formación integra del estudiante.

Haciendo énfasis en lo expuesto anteriormente al tema especificado, los docentes involucrados están preparados para aplicar estos nuevos cambios de transformación en el sistema educativo, con carisma y esa chispa de creatividad, partiendo del criterio de varios autores de lo que es Holo, que es el todo y la integración de las partes, porque esto va a ser el motor que va a mover la educación en el mundo. Dentro de este contexto se trabajará en conjunto con la participación de los estudiantes, padres de familia, empresas y directivos de la universidad.

En la sociedad de hoy, es importante en las empresas el uso de la innovación tecnología y holística en la contabilidad y gestión de costos, para conocer la información necesaria de forma rápida en la toma de decisiones, que lleven a una empresa al éxito, entre otras.

Es por eso que con el nuevo paradigma global, se ha considerado formar profesionales de excelencia que el país demanda para su desarrollo, por ello las universidades deben contar con 
Miguel Á. Jaime-Baque; Narda P. Merchán-Ponce; Amparo B. Baque-Morán; Marina B. Alvarado-Chancay; Erick G. Salazar-Ponce; Viviana del Rocío Saltos-Burí

información vigente y actualizada, así como lo exige la sociedad para dar respuestas pertinentes y acordes a la realidad. Es por esta razón, que se propone presentar la hipótesis, de que la aplicación de la Innovación holística y tecnológica en el aprendizaje de la contabilidad y la gestión de costos están estrechamente relacionada con el manejo de las empresas.

\section{Historia de la Contabilidad}

"Muchas de las cosas que se pueden contar no cuentan. Muchas de las cosas que no se pueden contar son las que cuentan"

\section{Albert Einstein}

Desde que el hombre formó sociedades comenzó el uso de la contabilidad, por la necesidad de almacenar provisiones de alimentos y llevar conteo de los mismos. En 1494 el monje Italiano Fray Luca Bartolomé Pacciolo publicó su obra llamada "SUMMA" que trataba sobre Algebra, Geometría, y Contabilidad, ya que consideró que ésta en su aplicación requiere del conocimiento matemático, por ello se le considera como el que nos brindó el legado de los fundamentos contables. (Valdivia, 2012)

Como en todos los campos, la contabilidad ha evolucionado, en respuesta a las necesidades sociales y económicas de la sociedad (inflación, globalización, modas, costumbres, uso medios electrónicos etc.). A medida que los negocios y la sociedad crecen y se vuelven más complejos a través del paso de los años, la contabilidad ha desarrollado y ha implementado nuevos conceptos, leyes, normas, y técnicas, de acuerdo con la tecnología moderna, para cumplir con las crecientes necesidades del mundo actual. 
Pero a pesar del gran desarrollo de operaciones mercantiles y la complejidad de negocios e instituciones y empresas estatales de hoy, los elementos básicos del sistema de partida doble continúan siendo los mismos.

El término Contabilidad proviene del latín "computare" que significa Contar. Incluye dos acepciones:

Narrar: Un sistema de Contabilidad debe ser narrativo en cuanto al orden cronológico de las tareas, trabajos y/o actividades.

Computar: En Contabilidad, todas las operaciones o transacciones deben registrarse en términos monetarios y expresarlas en cantidades.

La Contabilidad, sirve fundamentalmente para la toma de decisiones si no cumple con este objetivo, entonces pierde su esencia e integridad, es decir su razón de ser. Por otro lado, como la Contabilidad debe servir a inversionistas, clientes, empresarios, asesores, aseguradores, entre otros; es que existen diversos Tipos de Contabilidad. (Ídem).

A partir de esto, que el ser humano en su día a día en los albores de la humanidad, se dio cuenta de que era capaz de memorizar datos, de memorizar conceptos, fechas cantidades, pero llego al momento en el que se cuestiona esa capacidad, porque no es capaz de retener todo y es entonces cuando decide anotarlos, He aquí la frase, más vale un lápiz corto que una memoria larga, si en nuestro día a día decidimos que es mejor anotar las cosas y dejar para la memoria lo justo, mucho más importante es hacerlo en el mundo de la empresa, donde todo debe ser anotado o mejor dicho registrado contablemente, se puede decir que todo lo que ocurre en la empresa tiene una repercusión contable. (Ídem). 
La información contable juega un papel importante en la vida diaria de los negocios, a través de los resultados de la contabilidad los diferentes usuarios toman decisiones en beneficio de la empresa, de ahí la importancia de que dicha información cumpla ciertas características cualitativas como son relevancia y representación fiel.

Desde que el comercio existe en el mundo, que siempre ha tenido que ver alguna forma de que las personas contabilicen las compras, las ventas, los cobros, los pagos, esto siempre se ha tenido que llevar las cuentas de una forma u otra. Antiguamente se llevaba por el método de la partida simple, se llevaba unos listados, como podemos recordar en la historia.

La contabilidad, es conocerlo más a profundidad lo que esta genera en los negocios y en cualquier otra atividad que se desarrolle, es así, como se plasma un ideal tan relevante, porque no hay nada más importante que aprender a ser "persona", en toda la extención de la palabra. Desde este punto de vista se hace mención a una educación holística que mejora los resultados académicos, facilita la convivencia en el salón de clase, a yuda a los estudiantes a conocerse mejor consigo mismo y con los demás, es decir existe la integración y la participación de todos los involucrados, de esta manera, contribuye a despertar el sentido crítico y la conciencia ecológica, educa para el amor y la paz.

Partiendo de este enfoque se hace necesario conocer algunos conceptos, iniciando con lo que es innovación, holística y tecnología en la educación superior.

\section{Innovación.}

La palabra innovación proviene del sustantivo latino innovatio. Su étimo es novus, que constituye la base de un extenso campo léxico: novo, novitas, novius, renovo, renovatio, renovator, 
innovo e innovatio. Es interesante resaltar la existencia en latín del verbo novo (novare), sin prefijo, cuyo significado equivale al de los verbos innovar y renovar, (Rivas M. , 2003).

Es aquello que resuelve un problema, no queremos encontrar manera de destruir el planeta más rápidamente, ni hacer más profundo el consumirlo, la innovación entonces viene de un interés profundo y genuino por el bienestar de todos los seres a nivel mundial.

\section{¿Qué es Innovación Educativa?}

Revisando algunos conceptos, entre ellos está (Cañal, De León, 2002, 12 - 12), citado por (Rimari A. W., 2003, p. 3 - 4), menciona que la innovación educativa como:

“(un) conjunto de ideas, procesos y estrategias, más o menos sistematizados, mediante los cuales se trata de introducir y provocar cambios en las prácticas educativas vigentes. La innovación no es una actividad puntual sino un proceso, un largo viaje o trayecto que se detiene a contemplar la vida en las aulas, la organización de los centros, la dinámica de la comunidad educativa y la cultura profesional del profesorado. Su propósito es alterar la realidad vigente, modificando concepciones y actitudes, alterando métodos e intervenciones y mejorando o transformando, según los casos, los procesos de enseñanza y aprendizaje. La innovación, por tanto, va asociada al cambio y tiene un componente - explícito u oculto- ideológico, cognitivo, ético y afectivo. Porque la innovación apela a la subjetividad del sujeto y al desarrollo de su individualidad, así como a las relaciones teoríapráctica inherentes al acto educativo.”

Por su parte, (Imbernón, 1996, p. 64), afirma que: "la innovación educativa es la actitud y el proceso de indagación de nuevas ideas, propuestas y aportaciones, efectuadas de manera colectiva, 
Miguel Á. Jaime-Baque; Narda P. Merchán-Ponce; Amparo B. Baque-Morán; Marina B. Alvarado-Chancay; Erick G. Salazar-Ponce; Viviana del Rocío Saltos-Burí

para la solución de situaciones problemáticas de la práctica, lo que comportará un cambio en los contextos y en la práctica institucional de la educación”. (Ibídem, p. 4).

De otro lado, Juan Escudero (Pascual, 1988, p. 86), señala que: "Innovación educativa significa una batalla a la realidad tal cual es, a lo mecánico, rutinario y usual, a la fuerza de los hechos y al peso de la inercia. Supone, pues, una apuesta por lo colectivamente construido como deseable, por la imaginación creadora, por la transformación de lo existente. Reclama, en suma, la apertura de una rendija utópica en el seno de un sistema que, como el educativo, disfruta de un exceso de tradición, perpetuación y conservación del pasado. (...) innovación equivale, ha de equivaler, a un determinado clima en todo el sistema educativo que, desde la Administración a los profesores y alumnos, propicie la disposición a indagar, descubrir, reflexionar, criticar... cambiar." (Ibídem, p. 4).

Escudero concluye afirmando que hablar de innovación educativa significa referirse a proyectos socioeducativos de transformación de nuestras ideas y prácticas educativas en una dirección social e ideológicamente legitimada, y que esa transformación merece ser analizada a la luz de criterios de eficacia, funcionalidad, calidad y justicia y libertad social.

\section{Innovación en la Educación Superior}

A propósito de las nuevas tecnologías, cabe mencionar que su incorporación al ámbito educativo promueve la creación de nuevos perfiles para los actores del proceso de enseñanzaaprendizaje; el alumno debe preocuparse más por el proceso que por el producto, debe estar preparado para la toma de decisiones y debe ser capaz de elegir su ruta de aprendizaje. 
En definitiva, preparado para el autoaprendizaje lo cual abre un desafío a nuestro sistema educativo, preocupado durante mucho tiempo por la adquisición y memorización de información. Por su parte el profesor debe ser el elemento mediador, generador y organizador de las situaciones de aprendizaje.

La innovación supone una transformación, un cambio cualitativo significativo respecto a la situación inicial en los componentes o estructuras esenciales del sistema o proceso educativo. La innovación supone, también, partir de lo vigente para transformarlo. Por lo tanto, parte de un cambio en las estructuras y concepciones existentes. (Rimari A. W., 2010)

Cuando nos preguntamos sobre el objeto a innovar, las respuestas pueden ser muchas y tan generales o especificas como queramos, y pueden ir desde prácticas educativas vigentes, procesos formativos, recursos humanos y materiales hasta gestión institucional, el currículum y la enseñanza que incluya actitudes. Las circunstancias para que se dé la innovación educativa pueden ser de muy variada índole. Hay mayores requerimientos externos junto a más dificultades internas que reclaman como respuesta, cambios, modificaciones e innovaciones. (Rivas N. M., 2000)

La innovación suele responder a una necesidad o problema que regularmente requiere una respuesta integral; puede seguir un modelo centrado en la resolución de problemas y debe de ser impulsada por una gestión democrática, (Barraza, 2007, p.45). Y no debemos olvidar que el criterio básico de una experiencia innovadora es la participación crítica de los que la van a llevar a cabo. 


\section{Holismo}

El holismo es una manera de conceptualizar la realidad. Desde esta visión, cada vez que abordamos un fragmento de la realidad lo consideramos un todo, que parte de un todo mayor, una globalidad, e interactuamos en consecuencia. (Wernicke, S/f.)

La palabra holismo, acuñada por Jan Smuts en 1926 (Sudáfrica), proviene del griego holos, totalidad. En el mundo moderno, sin embargo, ya en el siglo XIX se hablaba con este mismo sentido de estructuras. También en los años veinte y treinta del siglo XX surgieron los términos sistema (von Bertalanffy, Alemania) y sistema funcional (Anokhin, Rusia). (Ídem).

En la historia, la humanidad comenzó conceptualizando holísticamente. Hace unas pocas centurias, con la aparición de la racionalidad como eje impulsor del desarrollo humano y su producto, la ciencia, la fragmentación adquirió enorme importancia, y el foco pasó a establecerse más en los fragmentos de cada realidad que en la realidad misma. Del holismo se pasó al fragmentarismo. (Ídem).

Éste último, qué duda cabe, representó un gran avance. No obstante, arrojó un subproducto inesperado: Muchos estudiosos de fragmentos de la realidad tomaron a sus recortes por los más importantes, y confundieron a sus fragmentos muy bien investigados con la realidad toda. Del fragmentarismo se pasó así a la especialización, un excelente paso lamentablemente muchas veces teñido de esta enorme dificultad: Creer que lo que existe es lo que el observador ve con la sola lupa que ha aprendido a sostener ante sus ojos. (Ídem).

El holismo intenta superar esta situación. La visión holística considera al universo y sus componentes (a cada "universo" parcial, por consiguiente también a cada ser humano) un sistema en 
que los elementos componentes interactúan entre sí. La observación de estas interacciones varía según el punto de mira del observador, según su propia lupa.

Un paradigma puede definirse como una conceptualización básica subyacente a un grupo de conocimientos que guía de modo duradero el desarrollo de ese mismo grupo de conocimientos (tanto se trate de ciencia, arte, pedagogía, economía, etc.). Puede hablarse en consecuencia de un paradigma holístico. (Ídem).

Sostienen que una visión holística es:

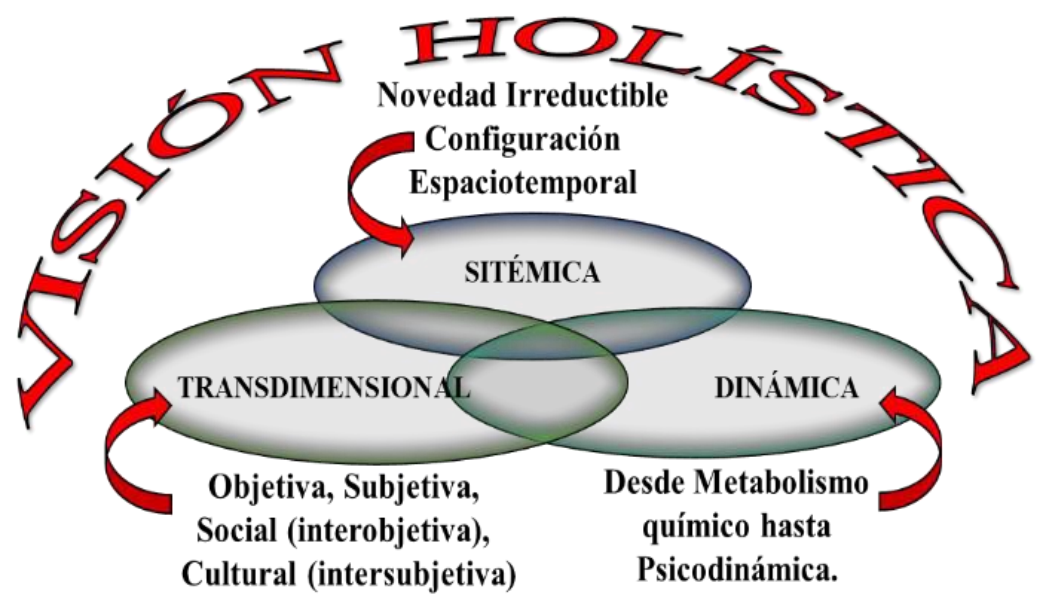

\section{Grafico 1 Visión Holísica. Fundación Holismo}

El ser humano es un sistema abierto en desarrollo. Llega al mundo (es concebido) con necesidades primordiales, a partir de cuya (in)satisfacción el ser humano inscribe sentimientos básicos, sobre los cuales construirá lógicas personales con las que procurará captar y comprender la realidad. Trae al mundo potencialidades que, al ser estimuladas por el Vcontexto cultural en que le toca vivir, producen un determinado desarrollo perceptual. A cada instante, la actividad de cada ser humano es producto de su terreno en continua interacción con su contexto. Esta actividad va registrándose en el individuo y en la cultura a modo de mapas perceptuales de fragmentos, 
Miguel Á. Jaime-Baque; Narda P. Merchán-Ponce; Amparo B. Baque-Morán; Marina B. Alvarado-Chancay; Erick G. Salazar-Ponce; Viviana del Rocío Saltos-Burí

interactuantes entre sí, modificando a cada momento el mapa global, general, integral, representativo de la realidad para cada individuo en particular. (Wernicke, S/f.).

En resumen, los supuestos básicos del paradigma holístico son:

La realidad se organiza en sistemas-holones, y así también el ser humano.

Es constante y obligada la interacción dinámica entre los elementos de un sistema, y así también del ser humano.

El ser humano ha sido históricamente descripto e interpretado simultáneamente desde diversas dimensiones (físico-molecular, biológica, emocional, cognitiva, espiritual) por diferentes observadores con diferentes lupas. En verdad se trata de un sistema transdimensional.

El ser humano se desarrolla constantemente desde su concepción, a partir de potencialidades propias y de acuerdo con las ofertas estimulatorias del contexto.

De tal modo, el ser humano va construyendo mentalmente un sistema de mapas fragmentarios, como forma de captación y organización de la realidad, en constante interacción y conformando finalmente un mapa global de su realidad.

Un enfoque sistémico-dinámico-transdimensional tendrá indudables consecuencias sobre la educación, la salud y la acción social.

El holismo es una visión integral. No es lo mismo que una visión integradora: No soy alguien que está formado por partes que se han amalgamado. La integración se inicia en un análisis e intenta llegar a una síntesis. (Wernicke, S/f.). 


\section{¿Qué es la Educación Holística?}

"El objetivo de la educación holista es ser el conducto a través del cual la conciencia humana pueda evolucionar hacia estadios de mayor integridad, de mayor profundidad, de mayor trascendencia". (Gallegos, 2008, p.149)

La educación holista es hoy un movimiento educativo mundial cuya generalización comenzó en la década de los 90's. La educación holista es un nuevo paradigma educativo, es una respuesta a la educación mecanicista basada en la superstición del materialismo, el reduccionismo y la fragmentación, es la nueva propuesta educativa para el siglo XXI, basada en un profundo sentido espiritual de la vida en el cosmos, no se reduce a ser un método educativo, ni se limita al ámbito escolar formal, es un modelo que define a la educación como un proceso de evolución de la conciencia, redefiniendo la totalidad del campo educativo. (Flake, , 1993).

"La educación holista es una pedagogía del amor universal, una llamada a vivir conscientemente en la compasión incondicional, un proceso para nutrir lo mejor del espíritu humano, un aprendizaje integral de los diferentes niveles de realidad, un conducto para la evolución de la conciencia, una educación del Ser, un diálogo para avivar el despertar de la genuina inteligencia: es el conocimiento directo de nuestra verdadera naturaleza" (Gallegos, Nava, , 2003)

La educación holista es un estado de conciencia, una visión integral de la vida, una llamada a vivir en el amor universal, una actitud compasiva, una apertura incluyente a la diversidad, un sendero de paz, diálogo y fraternidad; es el reconocimiento del amor universal como realidad educativa fundamental. Esta definición fue dada por Ramón Gallegos Nava, fundadores de la Fundación Internacional para la Educación Holista, quien ha creado el modelo de la educación 
Miguel Á. Jaime-Baque; Narda P. Merchán-Ponce; Amparo B. Baque-Morán; Marina B. Alvarado-Chancay; Erick G. Salazar-Ponce; Viviana del Rocío Saltos-Burí

holista, una pedagogía del amor universal que está enriqueciendo y nutriendo la vida de aquellos que buscan una educación con rostro humano, que vaya más allá del entrenamiento de la racionalidad instrumental. (Gallegos, Nava, , 1999)

La palabra holística tiene su origen en el vocablo griego holos, término utilizado para referirse a; todo, entero, integro, total o completo. La holística es un concepto que hace referencia a aquello que pertenece al holismo, corriente que considera a un sistema como un ente que trasciende a la agrupación de sus componentes, debido a que éste se comporta de modo diferente a la suma de sus partes, por lo tanto debe ser visto como un todo, que en vez de ser condicionado por el trabajo en conjunto de sus partes, éste determina el funcionamiento de sus componentes.

Cabe mencionar la interdependencia que existe entre componentes y el sistema, los cuales interactúan constantemente, dando lugar a acontecimientos que generan nuevas relaciones y eventos, provocando así una perspectiva integral, que permite apreciar aspectos que no se perciben al analizarlos por separado. Esto es de gran ayuda en el ámbito empresarial. (Domínguez, 2011).

\section{Pedagogía Holística}

El holismo, como movimiento educativo, se interesa por la interrelación entre lo físico, lo biológico, lo emocional, lo espiritual y lo intelectual, y la influencia recíproca entre estas dimensiones del ser. El holismo interactúa de forma global, es decir, con todas las dimensiones simultáneamente. Esto significa que toda experiencia modifica sus moléculas, su biología, sus emociones, su espíritu y su intelecto. (Corporación Hontanares, 2017)

La educación holística entonces, es la que aborda el desarrollo del ser desde su globalidad, teniendo en cuenta sus múltiples manifestaciones. Es la ciencia de educar para ser feliz, para amar, 
para utilizar correctamente las inteligencias sin conflictos y trasmitir el conocimiento de modo que pueda ser asimilado, comprendido y aplicado. Toma como punto de partida el reconocimiento de las inmensas potencialidades de cada ser humano y de su interés natural por su desarrollo. Requiere que el profesor tome en cuenta los conocimientos y experiencias del alumno, para que, partiendo de ellos, se pueda invitar al estudiante a pasar de lo conocido a lo que se quiere que aprenda. Otorga especial atención al desarrollo de los sentidos pues ellos le aportan al alumno elementos naturales para la comprensión y la síntesis de lo que vive y aprende. (Ídem).

La educación holística no es algo nuevo. Siempre ha estado presente en las filosofías orientales y comienza a ser reconocido en occidente a partir 1762, con la obra El Emilio de J. J. Rousseau. Otros pedagogos que señalan el holismo como principio fundamental de la educación son: Pestalozzi, Dewey, Steiner, Montessori, Rogers, Neill, entre otros.

Como afirma Carlos G. Wernic (Educación Holística Revista Educación Hoy 1994 n 10, Montevideo, Uruguay.), la educación holística implica un cambio dinámico:

- De enfatizar el desarrollo curricular a enfatizar el desarrollo humano

- De establecer estándares comunes para todos los educandos a reconocer y desarrollar las capacidades y los talentos.

- De organizar controles externos y reglas inflexibles a modelar y estimular la autodisciplina, el respeto y el amor mutuo.

- De usar evaluaciones "objetivas" (pruebas normatizadas) a desarrollar modelos de evaluación cooperativa. 
Miguel Á. Jaime-Baque; Narda P. Merchán-Ponce; Amparo B. Baque-Morán; Marina B. Alvarado-Chancay; Erick G. Salazar-Ponce; Viviana del Rocío Saltos-Burí

- De colocar a los padres en un rol periférico a considerar a los padres pares por completo activos.

- De crear ambientes de aprendizaje aislados a usar la comunidad global y local a fin de incorporar y asegurar aprendizajes de por vida.

De percibir a los docentes como técnicos que administran un curriculum rígido a considerar al docente un recurso y un facilitador que responde al potencial de cada estudiante. (Wernicke, S/f.), citado por (Corporación Hontanares, 2017).

\section{Tecnologia en la educación}

«Hemos pasado bruscamente de la edad de la piedra a la del circuito. Mientras que la rueda transporta simplemente los apoyos y los datos, el circuito establece relaciones entre los datos, y es el factor de la retroacción. De una época de transmisión pasamos a una era deretroacción, que es así mismo participación».

Marshall Macluhan (1911-1980)

La originalidad no está en lo fantástico, sino en el nuevo uso de cosas conocidas»

John Dewey (1859-1952)

La tecnología de la educación es la suma total de las actividades que hacen que la persona modifique sus ambientes externos (materiales) o internos (de comportamiento). Una aplicación sistemática de los recursos del conocimiento científico del proceso que necesita cada individuo para adquirir y utilizar los conocimientos. (Martinez - Salanova, 2017) 
Los últimos diez años han sido extraordinariamente fecundos en avances tecnológicos aplicables a la educación para poder ofrecer lo mejor y lo más importante en experiencias para los alumnos y hacer esto extensivo a un número cada vez mayor. Está absolutamente comprobado que el uso de los «multimedia» mejora el aprendizaje de los alumnos y al mismo tiempo reduce el tiempo de instrucción y los costos de la enseñanza. (Ídem).

Los alumnos necesitan para su futuro profesional de la utilización de los medios tecnológicos, ya que varían enormemente en su habilidad de percepción y aprendizaje; por lo tanto, en los requerimientos didácticos individuales. Algunos aprenden fácil y rápidamente a través de informaciones orales o impresas y con un mínimo de experiencias más directas. La mayoría requiere experiencias más concretas que incluyan los medios audiovisuales.

Hay muchos factores culturales que afectan el aprendizaje; por tanto los alumnos necesitan de una amplia gama de experiencias que incluya aspectos reales, representaciones visuales y símbolos abstractos. Las nuevas necesidades y expectativas laborales que el alumno tiene aconsejan una mayor participación del mismo en el aprendizaje mediante los métodos activos de investigación y experimentación. (Ídem).

Los programas educativos necesitan ser apreciados en términos de eficacia y flexibilidad de aplicación en cuanto a tiempo, personal y recursos de que se disponga. La demanda de empleo, exige una preparación que obliga al conocimiento de todo aquello que tiene que ver con la sociedad de la información, las nuevas tecnologías, la multiplicidad y variación profesional, la interacción de recursos, y en fin, de todo aquello que facilita la inserción laboral y profesional. (Ídem).

Los profesores, utilizando las nuevas tecnologías, pueden liberarse para realizar trabajos de orientación. Los nuevos patrones didácticos en los que se tiene en cuenta las nuevas tecnologías y 
Miguel Á. Jaime-Baque; Narda P. Merchán-Ponce; Amparo B. Baque-Morán; Marina B. Alvarado-Chancay; Erick G. Salazar-Ponce; Viviana del Rocío Saltos-Burí

los medios de comunicación para mejorar el aprendizaje suponen nuevas funciones de los profesores. No es el profesor el que debe proporcionar toda la información, ya que esta se puede presentarse más eficazmente por los medios apropiados, ya sea para proporcionarla a grandes grupos o para que cada alumno la amplíe por sí mismo en forma individual o para ser usada en un pequeño grupo de discusión. Los profesores ya como individuos, ya como equipos se encuentran liberados de trabajos rutinarios y pueden hacer el trabajo verdaderamente profesional y creativo, la orientación de los alumnos que hasta ahora se habían descuidado. Este trabajo de orientación y guía supone: amplia participación en la planificación y producción de materiales audiovisuales para adecuarlos a las necesidades de los grupos o de los individuos a que van destinados. (Ídem).

El uso de la tecnología para mejorar la comunicación obliga a cambiar los métodos rutinarios por otros más ágiles para alcanzar las metas educativas. La gran resistencia del personal docente a estos cambios se debe a que, el romper con la rutina perturba una situación habitual. También, inexactamente se cree que los nuevos medios deshumanizan la enseñanza, desplazan a los profesores y que la educación en consecuencia se hará autómata sin la calidad humana que le da la comunicación entre profesor y alumno.

En la actualidad, con el aumento de las disciplinas de estudio, del contenido de las mismas, la especialización y el aumento de la demanda profesional, lo que realmente sucede y de lo que generalmente se olvidan los educadores, es de que la verdadera humanización y el sello personal sólo pueden conseguirse con el uso adecuado de los recursos tecnológicos, que liberan al profesor del trabajo rutinario y de la mera transmisión de información, para permitirle realizar el trabajo de orientador y guía en la formación de sus alumnos. (Ídem). 


\section{Contabilidad y Gestión de Costos}

La contabilidad y gestión de costos, se ha vendio limitando al sistema de registros y a una forma de representación de tipo lineal que no permite utilizar los avances del siglo actual. Esta solo se limita a cálculos de la utilidad de un negocio y a definir el precio de un bien o servicio, en lugar de brindar alternativas de innovación, holísticas y tecnológicas aplicadas a la medición de variables de tipo financiero, económico y social. El mundo de las empresas y negocios el día de hoy se presenta complejo y con altas exigencias de sostenibilidad, competitividad y efectividad.

(Gandía, Montagud, \& Calabor, 2008), en el modelo aplicado contempla la Contabilidad de Costos, a partir de un proceso de aprendizaje integrado por tres fases: iniciación, profundización y concienciación, teniendo en cuenta las premisas del aprendizaje constructivista. Porporato (2015) realiza un estudio de caso aplicando el sistema de contabilidad de gestión basándose en la teoría de los costos de transacción se enfocó en determinar el impacto sobre el desempeño organizacional que tiene el propósito de usarlo y que los diferencie según como sean usados, para coordinar entre agentes y principales o para que el principal controle al agente. (Latorre, Aizaga, 2016).

(Gandía \& Montagud, 2011) y (Chanabá, 2014), presentan un abarcador e ilustrativo estudio empírico de la enseñanza, las innovaciones docentes y los resultados alcanzados en el aprendizaje. Este autor se dedicó a escribir una colección de tres libros sobre Contabilidad de Costos (Chanabá, 2010), que sirven de base a la enseñanza didáctica con sus enfoques de competencias, adorda temas actualizados y en consonancias con sus normas y disposiciones jurídicas nacionales. Representan fuentes de conocimiento y manejo imprescindibles para la formación de contadores. (Ídem). 
Miguel Á. Jaime-Baque; Narda P. Merchán-Ponce; Amparo B. Baque-Morán; Marina B. Alvarado-Chancay; Erick G. Salazar-Ponce; Viviana del Rocío Saltos-Burí

En correspondencia con la evolución, (Fernández, 2000), sintetiza que la contabilidad de costos ha evolucionado en los últimos años surgiendo nuevos enfoques que toman en cuenta los causales de costos y nuevas técnicas de costeo de productos bajo el enfoque estratégico, tales como el método del costeo basado en actividades y el enfoque de la cadena de valor, el cual es considerado como una herramienta útil a la gerencia estratégica. (Ídem).

Algunos aspectos que constituyen brechas de profundización para estudios posteriores, y (González, Camacho, \& Sangerman, 2011), identifican como aspectos necesarios a incluir, son las actividades económicas y sociales, que alcanzan en ocasiones cifras cuantiosas y deben incluirse en el estudio de los procesos económicos actuales. Realizan un análisis sugerente de estos elementos que consideran no son tenidos en cuenta en la contabilidad de costos privados de las empresas. (Ídem).

La contabilidad y la gestión de costos, son dos elementos prioritarios y muy esenciales en la utilización de instrumentos para el seguimiento de costos de las actividades desarrolladas por un ente productivo, es decir son herramientas de gran valía para el desarrollo de modelos de gestión y muy útiles para el éxito de los negocios, cuando hablamos de gestión de costos estamos refiriéndonos a un sistema complejo con un carácter esencialmente etratégico con una visión holística y tecnológica.

La Contabilidad es la ciencia que evoluciona y que tiene como objetivo principal clasificar, medir y valorar las operaciones financieras realizadas por la empresa, basada en conocimientos razonados y lógicos para registrar, sintetizar y analizar los resultados, obteniendo una base informativa adecuada para su gestión y toma de decisiones en el contexto externo e interno de la empresa. 
Los cambios son significativo cada vez más, los profesionales de hoy deben ser expertos con excelentes habilidades sobre Contabilidad y gestión de costos, necesitan estar preparados para desenvolverse en cualquier ámbito de trabajo, como: asesores de negocios; analistas financieros; comunicadores excelentes; negociadores capaces y administradores de primera clase. Al mismo tiempo, integridad, objetividad y voluntad de adoptar una postura firme. Teniendo en cuenta que la evolución de la Contabilidad y Gestión ha estado relacionada con el desarrollo empresarial y organzacional.

\section{Rol de la Inovacion, Holística y Tecnológica en la Contabilidad y Gestión de Costos en la}

\section{Educación Superior}

La ciencia y los enfoques tecnológicos han dado un giro de cambio y transformación, permitiendo a la contabilidad planear y fortalecer el ejercicio profesional. Es por eso que conociendo los retos que tienen que afrontar los profesionales en este nuevo siglo, se hace énfasis en proyectar nuevos conocimientos o avances científicos de la contabilidad o gestión de costos a través de la investigación.

Esta Contabilidad es tecnológica porque aplica tecnologías debidamente probadas, y se basa en el modelo sistémico, holístico e helicoidal. Asimismo, los Tipos de Contabilidad Tecnológicos como el Benchmarking nos sirven para comparar los resultados obtenidos con el mejor de la competencia y la Contabilidad Virtual o la Contabilidad On-line, que utilizan tecnología innovadora, donde la rapidez, es su mayor fortaleza. (Valdivia, 2012).

La diferencia entre la resolución de problemas universitarios a la vida profesional radica en su nivel de exigencia, pero también con relación a la complejidad, que sería afrontada con éxito si es que se adopta un pensamiento holístico. 
Miguel Á. Jaime-Baque; Narda P. Merchán-Ponce; Amparo B. Baque-Morán; Marina B. Alvarado-Chancay; Erick G. Salazar-Ponce; Viviana del Rocío Saltos-Burí

Muchas veces una solución sencilla para un problema complejo puede funcionar en un corto plazo, pero a mediano y largo plazo puede generar otros tipos de conflictos que se podrían visionar si es que consideramos una perspectiva holística de las cosas.

Según Fuentes, citado por (Carvajal, 1998, p.5) “Todos están de acuerdo en que la naturaleza del progreso del siglo XXI dependerá, ante todo, del factor educativo. La educación como base de conocimiento. El conocimiento como base de información. La información como base del desarrollo" [sic]. Lo que se necesita es pasar de políticas educativas de gobiernos a verdaderas políticas de Estado que respondan a necesidades nacionales y regionales, respaldadas como garantía de sostenibilidad, en donde lo importante es el conocimiento compartido a través de la investigación.

Parafraseando lo manifestado por (Casal \& Vilorio, 2007, p. 24). La enseñanza de la contabilidad o gestión de costos desde la innovación holística y lo importante de la tecnología, es afrontar los desafíos. Vivimos en un mundo cambiante; no existe ninguna razón para considerar que la contabilidad pueda estar ajena a los mismos. Los grandes desarrollos tecnológicos, matemáticos y científicos se encuentran trabajando para ejercer un impacto en la práctica y en la teoría contable, y eso es una realidad que no puede ser ignorada ni por los académicos (profesores del área contable), ni por quienes practican el oficio de la contabilidad (contadores públicos). Esto impone un replanteamiento de la estructura de la enseñanza en contabilidad, que satisfaga tanto las necesidades presentes como las futuras. (Mattessich, 2002).

¿Qué es la Holística Empresarial?

La empresa es un sistema que busca satisfacer la demanda de un bien o servicio, para lograrlo, debe de contar entre otras cosas, con elementos humanos, que son indispensables para 
llevar a cabo las tareas que permitirán materializar el ideal de la empresa. Al formar parte de un todo; el capital humano debe estar en óptimas condiciones si se pretende que la empresa opere de manera correcta.

Durante mucho tiempo se pensó que la mejor manera de optimizar el desempeño de una empresa era; dividir las responsabilidades, he incluso hacer subdivisiones a estas para simplificar las actividades, sin embargo esto ha traído consecuencias negativas para las empresas, ya que al responsabilizar al individuo a una pequeña parte del sistema, se provoca un sentimiento de no identificación con el resto de las operaciones, debido a que se desconoce la consecuencia del trabajo realizado, así como la importancia de éste para el sistema.

Lo anterior aísla y desmoraliza al individuo, que a su vez pierde el gusto por su actividad. La holística empresarial busca integrar equipos de trabajo, bajo una cultura que haga al empleado sentirse parte de la empresa, promoviendo la superación personal y una mejor calidad de vida, sin dejar de lado la responsabilidad con el ambiente y la sociedad, de tal manera que se produzca una sinergia, capaz de sumar experiencias y conocimientos que darán como resultado; un nuevo sistema listo para enfrentarse a los cambios de manera sostenible. (Domínguez, 2011).

Una vez que la empresa es considerada como un todo, que se encuentra íntimamente relacionado con su capital humano, se estará llevando la actividad empresarial a otros niveles de eficiencia. Esto trae consecuencias económico-financieras favorables por la ley de causa y efecto. Debido a que las empresas son el motor de nuestra sociedad, al implementar la holística empresarial se obtienen beneficios que van más allá de lo superfluo, el beneficio es total para nuestra sociedad como seres humanos. (Ídem). 
Miguel Á. Jaime-Baque; Narda P. Merchán-Ponce; Amparo B. Baque-Morán; Marina B. Alvarado-Chancay; Erick G. Salazar-Ponce; Viviana del Rocío Saltos-Burí

Hoy en día lo único seguro es el cambio. Nada permanece constante. Cambio no es una palabra de moda, es una realidad. El cambio es un proceso natural implícito en todas las formas de vida. Nuestro entorno evoluciona, nada permanece estático.

La Educación para la innovación y la tecnología se apoya en el aprendizaje consiente, que es la principal herramienta de la educación holística, para abrir la posibilidad en la educación superior con los cambios de transformación en el interaprendizaje de la contabilidad y gestión de costos en la educación superior. En otras palabras, es el engranaje de conocimientos para ser potencializados con una visión integral, de esta manera el estudiante se siente inspirado para trabajar y construir un mundo mejor.

En la siguiente gráfica se muestra la integración

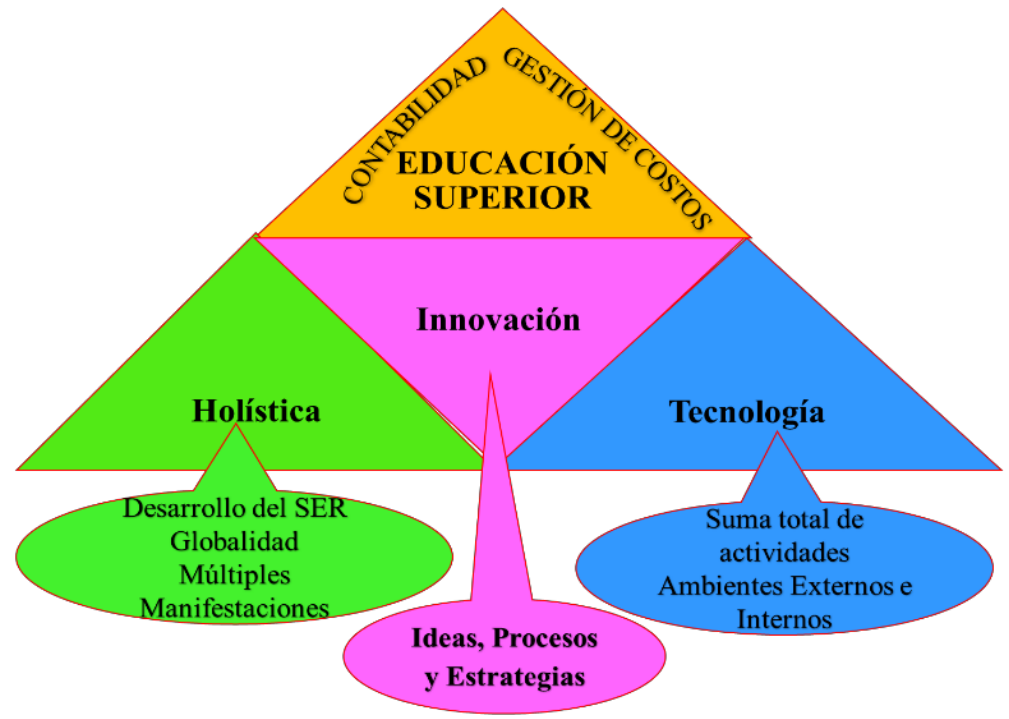

Grafico 1 Visión Holísica. Fundación Holismo 


\section{Metodología}

Este artículo, es resultado de una investigación de enfoque cualitativo, basada en una serie de trabajos públicados de gran relevancia sobre los cambios de transformación en la educación superior, desde la innovación, la holística y la tecnología, que son herramientas estratégicas para el eficiente trabajo docente educativo.

Es decir se trata de una revisión documental de la temática de tipo exploratorio, muestra la situación que prevalece en la educación, así como la tecnología y la innovación holística que es una verdadera oportunidad para que la educación superior, las incluya como una herramienta estratégica en el trabajo académico. Para satisfacción compleja desde el enfoque metodológico de la investigación holística, es realizar la tarea con creatividad e innovación para potencializar el mundo moderno.

\section{Conclusiones}

El artículo de la Contabilidad requiere establecer una estructura jerárquica con un orden conceptual que oriente teórica y operativamente a los actores relacionados con los procesos y resultados de la actividad contable.

Partiendo de esto es, que el ser humano es una entidad multidimensional con una corresponsabilidad social de manera lógica, es aquí donde la educación que se impartirá tiene que poseer una visión holística, innovadora y con aplicación tecnológica del estudiante. La educación a nivel mundial requiere el desarrollo cognitivo, por lo que la formación del educando debe elevar su potencial en todas sus dimensiones además debe desarrollar competencias para la vida. 
Miguel Á. Jaime-Baque; Narda P. Merchán-Ponce; Amparo B. Baque-Morán; Marina B. Alvarado-Chancay; Erick G. Salazar-Ponce; Viviana del Rocío Saltos-Burí

Es por lo anterior expuesto en el desarrollo de competencias requiere no solamente de programas académicos con una visión holística, innovadora y tecnológica de la persona, sino de un compromiso de las instituciones educativas de educación superior, para garantizar la calidad de la enseñanza y, por consiguiente, del aprendizaje. En este sentido, es por eso que este documental del artículo de la contabilidad de gestión de costo en la Educación Holística es un todo en el trabajo educativo en todo el proceso.

\section{Referencias}

Barraza, M. A. (2007, p.45). Análisis conceptual del término innovación educativa, Universidad Pedagógica de Durango. Universidad Pedagógica de Durango, México.

Cañal, De León, P. o. (2002, 12 - 12). La Innovación Educativa, . Madrid.

Carvajal, L. (1998, p.5). “Transformar la educación para reconstruir la nación”. Ponencia presentada en el acto inaugural de la Asamblea Nacional de Educación. Caracas.

Casal , R., \& Vilorio, N. (2007, p. 24). La ciencia contable, su historia, filosofía, evolución y su producto. Red de Revistas Científicas de América Latina, el Caribe, España y Portuga. Obtenido de http://www.redalyc.org/pdf/257/25701503.pdf

Chanabá, J. A. (2010). Contabilidad de Costos. Quito: Edinum.

Chanabá, J. A. (2014). Reseña de la Colección: Contabilidad de costos. Revista Publicando, 1 (1), 86-89.

Corporación Hontanares. (2017). Pedagogía Holística. Hontanares, Habana - Cuba. Obtenido de http://www.hontanares.edu.co/index.php?option=com_content\&view=article\&id=10\&Itemid $=6$

Domínguez, C. (2011). Codigosdelser.com. Obtenido de http://www.codigosdelser.com/holisticaempresarial-articulos/que-es-la-holistica-empresarial/

Flake, , C. L. (1993). (1993) Holistic Education: principles, perspectives and practices. University of South Carolina. Holistic Education Press. Brandon.V.T.

Gallegos, Nava, , R. (2003). Pedagogía del Amor Universal: Una visión holista del mundo. México,. Obtenido de https://www.simplechange.com.mx/la-educacion-holista-en-mexico-2/ 
Gallegos, Nava, , R. (1999). Educación Holista, pedagogía del amor universal. Fundación Internacional para la Educación Holista. , Guadalajara.

Gallegos, N. R. (2008, p.149). Educación para la Vida y la Paz: Más Allá de la Calidad Educativa. Fundación Internacional para la Educación Holista, México. Obtenido de https://www.simplechange.com.mx/la-educacion-holista-en-mexico-2/

Gandía, J. L., \& Montagud, M. D. (2011). Innovación docente y resultados del aprendizaje: un estudio empírico en la enseñanza de la contabilidad de costes. Revista Española de Financiación y Contabilidad, XL (152; octubre-diciembre), 677-698.

Gandía, J. L., \& Montagud, M. D. (2011). Innovación docente y resultados del aprendizaje: un estudio empírico en la enseñanza de la contabilidad de costes. Revista Española de Financiación y Contabilidad, XL (152; octubre-diciembre),677-698.

Gandía, J. L., Montagud, M. D., \& Calabor, M. (2008). Entorno multimedia y telemático aplicado a la Contabilidad de Costes. @ tic. revista d'innovació educativa. (1), 26-31.

Gandía, J. L., Montagud, M. D., \& Calabor, M. (2008,). Entorno multimedia y telemático aplicado a la Contabilidad de Costes. @ tic. Revista d'innovació educativa. (1),, p. 26-31.

González, A., Camacho, M., \& Sangerman, M. D. (2011). Incorporación de cuentas ecológicas y servicios ambientales en las matrices de contabilidad social. Revista Mexicana de Ciencias Agrícolas, 2 (5; 1 de septiembre-31 de octubre), , 715-731.

Imbernón, F. (1996, p. 64). En busca del discurso perdido. Buenos Aires-Argentina.: Edt. Magisterio del Río de la Plata.

Latorre, Aizaga, F. L. (2016). Estado del Arte de la Contabilidad de Costos. Revista Publicando, 3(8). 2016, 513-528 ISSN 1390-9304.

Martinez - Salanova, S. E. (2017). Educomunicación. Medios, recursos y nuevas tecnologías para la educación.". Edición on line. Obtenido de https://www.uhu.es/cine.educacion/cineyeducacion/salanova.htm

Mattessich, R. (2002). Contabilidad y Métodos Analíticos. Red de Revistas Científicas de América Latina, el Caribe, España y Portugal.

Pascual, R. (1988, p. 86). La gestión educativa ante la innovación y el cambio. Madrid.

Rimari, A. W. (2003, p. 3 - 4). La Innovación educativa. Lima - Perú.

Rimari, A. W. (2010). La innovación Educativa, instrumento de desarrollo. Obtenido de http://www.fundep.gob.pe/boletin/Innovación.pd

Rivas, M. (2003). La innovación educativa. Madrd: Edit. Síntesis. Obtenido de http://www.uaa.mx/direcciones/dgdp/defaa/descargas/innovacion_educativa_octubre.pdf 
Miguel Á. Jaime-Baque; Narda P. Merchán-Ponce; Amparo B. Baque-Morán; Marina B. Alvarado-Chancay; Erick G. Salazar-Ponce; Viviana del Rocío Saltos-Burí

Rivas, N. M. (2000). Innovación educativa. Teoría, procesos y estrategias. Obtenido de http://tecnologiaeducativaunefa.wikispaces.com/file/view/Innovaci\%C3\%B3n+Educativa+T eor\%C3\%ADa+Procesos+y+Estrategias+Manuel+Rivas+Marcano.pdf.

Valdivia, R. ,. (2012). Nueva clasificación mundial de tipos de contabilidad [todos los que existen actualmente en el mundo. QUIPUKAMAYOC Revista de la Facultad de Ciencias Contables, Vol. $20 \quad N^{\circ} \quad 37 \quad$ pp. $54-60 \quad$ (2012) UNMSM, . Obtenido de http://sisbib.unmsm.edu.pe/bibvirtual/publicaciones/quipukamayoc/2012/V20n35I/pdf/a06v35n1.pdf

Wernicke, C. G. (S/f.). Desde nuestro Enfoque Sistémico - Dinámico - Transdimensional: ¿Qué es Holismo? buenos Aires - Argentina: Fundación Holismo de Educación, Salud y Acción Social. Obtenido de http://www.holismo.org.ar/index.php/holismo/que-es-holismo 